\title{
Body Size and Colorectal Cancer Risk After 16.3 Years of Follow-up: An Analysis From the Netherlands Cohort Study
}

Citation for published version (APA):

Hughes, L. A. E., Simons, C. C. J. M., van den Brandt, P. A., Goldbohm, R. A., van Engeland, M., \& Weijenberg, M. P. (2011). Body Size and Colorectal Cancer Risk After 16.3 Years of Follow-up: An Analysis From the Netherlands Cohort Study. American Journal of Epidemiology, 174(10), 1127-1139. https://doi.org/10.1093/aje/kwr247

Document status and date:

Published: 15/11/2011

DOI:

10.1093/aje/kwr247

Document Version:

Publisher's PDF, also known as Version of record

Document license:

Taverne

Please check the document version of this publication:

- A submitted manuscript is the version of the article upon submission and before peer-review. There can be important differences between the submitted version and the official published version of record.

People interested in the research are advised to contact the author for the final version of the publication, or visit the DOI to the publisher's website.

- The final author version and the galley proof are versions of the publication after peer review.

- The final published version features the final layout of the paper including the volume, issue and page numbers.

Link to publication

\footnotetext{
General rights rights.

- You may freely distribute the URL identifying the publication in the public portal. please follow below link for the End User Agreement:

www.umlib.nl/taverne-license

Take down policy

If you believe that this document breaches copyright please contact us at:

repository@maastrichtuniversity.nl

providing details and we will investigate your claim.
}

Copyright and moral rights for the publications made accessible in the public portal are retained by the authors and/or other copyright owners and it is a condition of accessing publications that users recognise and abide by the legal requirements associated with these

- Users may download and print one copy of any publication from the public portal for the purpose of private study or research.

- You may not further distribute the material or use it for any profit-making activity or commercial gain

If the publication is distributed under the terms of Article 25fa of the Dutch Copyright Act, indicated by the "Taverne" license above, 


\title{
Original Contribution
}

\section{Body Size and Colorectal Cancer Risk After 16.3 Years of Follow-up: An Analysis From the Netherlands Cohort Study}

\author{
Laura A. E. Hughes*, Colinda C. J. M. Simons, Piet A. van den Brandt, R. Alexandra Goldbohm, \\ Manon van Engeland, and Matty P. Weijenberg \\ * Correspondence to Dr. Laura A. E. Hughes, Department of Epidemiology, Faculty of Health Medicine and Life Science, Maastricht \\ University, P.O. Box 616, 6200 MD, Maastricht, the Netherlands (e-mail: laura.hughes@ maastrichtuniversity.nl).
}

Initially submitted February 4, 2011; accepted for publication June 22, 2011.

A large body size may differentially influence risk of colorectal cancer (CRC) by anatomic location. The Netherlands Cohort Study includes 120,852 men and women aged 55-69 years who self-reported weight, height, and trouser/skirt size at baseline (1986), as well as weight at age 20 years. Derived variables included body mass index (BMI; weight $(\mathrm{kg}) /$ height $\left.(\mathrm{m})^{2}\right)$, BMI at age 20 years, and BMI change. After 16.3 years of follow-up (1986-2002), 2,316 CRC cases were available for case-cohort analysis. In men, the highest risk estimates were observed for body fat (per 5-unit increase in $\mathrm{BMI}$, hazard ratio $(\mathrm{HR})=1.25,95 \%$ confidence interval $(\mathrm{Cl}): 1.05,1.46$; for highest quintile of trouser size vs. lowest, $\mathrm{HR}=1.63,95 \% \mathrm{Cl}: 1.17,2.29(P$-trend $=0.02))$ and appeared more closely associated with distal colon tumors (for BMI (5-unit increase), $\mathrm{HR}=1.42,95 \% \mathrm{Cl}: 1.13,1.79$; for highest quintile of trouser size, $\mathrm{HR}=2.56,95 \% \mathrm{Cl}: 1.55,4.24(P$-trend $<0.01))$ than with proximal colon or rectal tumors. In women, body fat was not associated with $\mathrm{CRC}$ risk unless it was considered simultaneously with physical activity; a large trouser/skirt size and a low level of physical activity increased risk for all subtypes. Height was associated with risk of CRC, especially distal colon tumors (highest quintile vs. lowest: $\mathrm{HR}=1.53,95 \% \mathrm{Cl}: 1.03,2.27 ; P$-trend $=0.05$ ), in women only.

body height; body mass index; cohort studies; colonic neoplasms; rectal neoplasms; waist circumference

Abbreviations: BMI, body mass index; $\mathrm{Cl}$, confidence interval; CRC, colorectal cancer; HR, hazard ratio; NLCS, Netherlands Cohort Study.

There is convincing evidence that a large body size increases the risk of colorectal cancer (CRC) (1). CRC risk has traditionally been studied according to anatomic location in the colon or rectum. However, several anatomic, embryologic, and physiologic differences exist between subanatomic locations in the colorectal tract, and therefore arising tumors may have different etiologic pathways (2-5). Thus, it may be more rational to consider CRC risk at 3 different locations: the proximal colon, distal colon, and rectum. Case-control and prospective cohort data suggest that in both men and women, body mass index (BMI) is more strongly associated with tumors of the distal colon than with tumors of the proximal colon (6-13). Several studies have suggested that waist circumference, as a proxy measure for abdominal obesity, may be a better indicator of CRC risk than BMI $(14,15)$ and that adult attained height as a proxy measure for early-life nutritional and socioeconomic exposures is also a convincing risk factor for CRC (1). How other indicators of body size, such as waist circumference and height, might influence $\mathrm{CRC}$ risk at different tumor subsites is less clear.

At present, one can only hypothesize as to how indicators of body size may influence subsite-specific pathways of colorectal carcinogenesis. Approximately $85 \%$ of tumors are thought to develop via the traditional adenoma-carcinoma pathway, characterized by mutations in the KRAS oncogene and the $A P C$ tumor suppressor gene, as well as chromosomal instability (16). Such tumors are most frequently observed in the distal colon (3). The other $15 \%$ of tumors are thought to arise via the serrated neoplasia pathway, characterized in part by a high degree of epigenetic instability and microsatellite instability (16). Such tumors are most frequently observed in the proximal colon (17-19). It has been reported that adult BMI and indicators of abdominal obesity are associated more strongly with tumors that are not characterized by 
epigenetic instability (20-22). This suggests that adult body fat rather influences CRC risk via chromosomal instability and may explain the stronger associations observed between body fat and distal tumors.

Furthermore, timing of exposure may be important for defining pathways. It has been observed that a high BMI in early adulthood and a large BMI change from early adulthood to later adulthood are associated with a higher risk of CRC (13); that a high BMI at age 20 years is associated with a greater risk of having a tumor characterized by epigenetic instability (21); and that taller persons have a higher risk of tumors characterized by epigenetic instability (Hughes et al., Maastricht University, unpublished manuscript).

Here, we used data from a prospective study, the Netherlands Cohort Study (NLCS), to investigate the association between multiple indicators of body size and sex-specific risk of CRC, as well as the associations between these indicators and sex-specific CRC risk at specific tumor subsites, including the proximal colon, distal colon, rectosigmoid junction, and rectum. We considered BMI and trouser/skirt size as adult indicators of body fat, and we also considered adult attained height, BMI at age 20 years, and BMI change when assessing risk in order to study whether timing of exposure was associated with differential risk. We hypothesized that body fat would be associated more with tumors of the distal colon, whereas height might be associated more with proximal colon tumors.

\section{MATERIALS AND METHODS}

\section{Study population and design}

The NLCS includes 58,279 men and 62,573 women aged 55-69 years at baseline (1986) who completed a selfadministered questionnaire involving 150 food items, as well as questions on lifestyle and health. Municipal registries from throughout the Netherlands were used to constitute an efficient sampling frame (23). The NLCS uses a case-cohort approach for data processing and analysis; case subjects were derived from the entire cohort, and the number of person-years at risk for the entire cohort was estimated from a subcohort of 5,000 persons who were randomly sampled from the full cohort at baseline. All subcohort members who reported prevalent cancer (excluding skin cancer) at baseline were excluded from the analyses, leaving 4,654 participants. Further details of the NLCS design have been published elsewhere (23-25).

Incident CRC cases were identified by annual record linkage to 9 regional cancer registries and a national pathology database (PALGA) (26). The completeness of cancer followup in the NLCS is almost 100\% (27). CRCs were classified by anatomic location as proximal colon (International Classification of Diseases for Oncology, First Edition, codes 153.0, 153.1, 153.4, 153.5, and 153.6), distal colon (codes 153.2, 153.3, and 153.7), rectosigmoid (code 154.0), or rectal (code 154.1) tumors. Persons with incomplete or inconsistent baseline questionnaires were excluded. After additionally excluding persons who were missing data on variables adjusted for in the multivariate analysis, 3,197 subcohort members and 2,316 CRC cases remained. Among men, the numbers of tumors by location were 327 proximal colon, 427 distal colon, 33 unspecified colon, 125 rectosigmoid junction, and 299 rectum; for women, the numbers were 459 proximal colon, 327 distal colon, 27 unspecified colon, 87 rectosigmoid, and 205 rectum.

\section{Assessment of anthropometric variables, diet, and physical activity}

Height $(\mathrm{cm})$, body weight $(\mathrm{kg})$, and body weight at age 20 years $(\mathrm{kg})$ were self-reported on the baseline questionnaire. BMI was calculated as weight $(\mathrm{kg}) /$ height $(\mathrm{m})^{2}$. Participants were also asked to report their lower-body clothing (trouser or skirt) size from their clothing label (in Dutch sizes). Trouser/skirt size has been shown to be an adequate proxy measure for waist circumference when predicting cancer risk in the NLCS (28).

Both occupational physical activity in the longest-/last-held job and baseline nonoccupational physical activity were used to assess risk, depending on sex (Simons et al., Maastricht University, unpublished manuscript). For analyses of men, occupational physical activity was used. Participants were asked to report job title(s) and job duration(s) on the baseline questionnaire. Assessment of physical activity at work was based on the job held for the longest amount of time. Total energy expenditure was based on a rating system developed by Hettinger et al. (29). Men were classified into 3 energy expenditure groups: $<8 \mathrm{~kJ} /$ minute, $8-<12 \mathrm{~kJ} /$ minute, and $\geq 12 \mathrm{~kJ} /$ minute. For analyses with women, nonoccupational physical activity was used to assess risk, because most women of this generation had not held a job or had worked for only a short period of time. It was calculated by totaling the number of minutes per day spent cycling/walking to work, going to shops, and walking the dog and the number of hours spent per week engaging in gardening/odd jobs, recreational cycling/walking, and sports/exercise, as reported on the baseline questionnaire. Women were classified as having a low ( $<30$ minutes/day), intermediate (30-90 minutes/day), or high ( $>90$ minutes/day) level of physical activity.

\section{Statistical analyses}

Data were analyzed with Stata, version 10 (StataCorp LP, College Station, Texas), separately for men and women. Cox proportional hazards analysis using the case-cohort approach was used to estimate hazard ratios and 95\% confidence intervals for the association between total CRC risk and BMI (per 5-unit increase, in sex-specific quintiles), BMI at age 20 years (per 5 -unit increase, in sex-specific quintiles), BMI change $(<0,0-<4,4-<8$, or $\geq 8$ units; categories were based on a previous NLCS publication (30)), trouser/skirt size (per 2-unit size increase, in 5 sex-specific categories), and height (per 5-cm increase, in sex-specific quintiles). To test for a linear trend across categories, we used the median anthropometric variable within quintiles/categories as a continuous variable. Statistical significance was defined as $P<0.05$ (2-sided testing).

Multivariate models. We adjusted for potential confounders selected a priori from the literature and those that introduced more than a $10 \%$ change in hazard ratios. Selected a 
priori were age, family history of CRC (yes/no), smoking status (never smoker, ex-smoker, or current smoker), socioeconomic status (educational level), total energy intake (kcal/day), alcohol intake $(0,0.1-4,5-14,15-29$, or $\geq 30$ g/day), recreational physical activity $(<30,30-90$, or $>90$ minutes/day) for women, and occupational physical activity at the longestheld job ( $<8,8-<12$, or $\geq 12 \mathrm{~kJ} /$ minute) for men. No variables introduced more than a $10 \%$ change in the hazard ratios. BMI change was additionally adjusted for BMI at age 20 years, and height was additionally adjusted for body weight (30). We also considered a model for women that included additional adjustment for oral contraceptive use (ever, never), duration of oral contraceptive use (years), use of hormone replacement therapy (ever, never), and duration of hormone replacement therapy (years). This extra adjustment did not influence effect estimates, and results are not presented.

Interaction with physical activity. Evidence suggests that among persons with lower physical activity, BMI becomes a more important indicator of colon cancer risk (9) and that underlying population levels of physical activity can impair or enhance the ability to identify colon cancer associations with other risk factors (31). We created a 2-way interaction between trouser/skirt size and physical activity. Categories of physical activity were based on self-reported recreational physical activity (women) and occupational physical activity (men), and trouser/skirt size was dichotomized on the basis of sex-specific median size. We used trouser/skirt size because evidence suggests that fat distribution is more important than body weight or BMI for CRC risk, especially in women (14). We also considered this interaction for colonic and rectal tumors separately.

For all analyses, the proportional hazards assumption was tested using scaled Schoenfeld residuals and visual inspection of the hazard curves. Furthermore, we repeated the analyses according to duration of follow-up by splitting the follow-up time at 8 years ( $<8$ years vs. $\geq 8$ years). To account for the additional variance introduced by sampling the subcohort from the entire cohort, we estimated standard errors using the robust option.

Tests for heterogeneity. We conducted tests for heterogeneity to evaluate differences between sublocalizations of tumors (e.g., proximal colon vs. distal colon), using the competing-risks procedure in Stata. However, the standard error for the difference in the log hazard ratios from this procedure assumes independence of both estimated hazard ratios, which would overestimate that standard error and thus overestimate the $P$ value for their difference. Therefore, we estimated these $P$ values and the associated confidence intervals on the basis of a bootstrapping method that was developed for the case-cohort design, as described previously (32). Each bootstrap analysis was based on 1,000 replications.

\section{RESULTS}

\section{Descriptive characteristics}

For both men and women, a higher trouser/skirt size corresponded with increasing BMI, and total energy intake was highest in the third quintile of BMI. Furthermore, the great- est proportion of persons with a university-level education was observed in the lowest quintile of BMI, as was the greatest proportion of current smokers. In men, levels of occupational physical activity were relatively similar across quintiles of BMI, whereas in women, higher levels of recreational physical activity were reported by persons in the lowest quintile of BMI (Tables 1 and 2).

\section{Associations with overall CRC in men}

Age-adjusted results are not presented, since they were comparable with multivariate results. Associations between body size and CRC risk in men are presented in Table 3. With respect to body fat, BMI modeled per 5-unit increase was associated with total CRC (hazard ratio $(\mathrm{HR})=1.25$, 95\% confidence interval (CI): 1.05, 1.46). When BMI was modeled in quintiles, associations did not reach statistical significance. Stronger associations were observed with respect to trouser size; a statistically significant association was observed when comparing persons with the largest trouser size to those with the smallest size (HR $=1.63,95 \%$ CI: 1.17 , 2.29; $P$-trend $=0.02$ ).

With respect to BMI at age 20 years, BMI change, and height, associations with overall CRC did not reach statistical significance.

\section{Associations by tumor subsite in men}

All indicators of body fat appeared to be most strongly associated with tumors of the distal colon. Increasing BMI (per 5 units) was associated with both distal ( $\mathrm{HR}=1.42,95 \%$ CI: $1.13,1.79)$ and rectosigmoid (HR $=1.49,95 \% \mathrm{CI}: 1.05$, 2.11) tumors. A statistically significant trend was observed over quintiles of BMI for these two subsites $(P=0.05)$. BMI at age 20 years was associated only with tumors of the distal colon (5-unit increase: HR $=1.09,95 \%$ CI: 1.00, 1.19; highest quintile vs. lowest: $\mathrm{HR}=1.47,95 \% \mathrm{CI}: 1.03,2.08$ $(P$-trend $=0.05))$. With respect to a positive BMI change, there was a suggested dose-response association for tumors of the distal colon $(P$-trend $=0.05)$. The strongest association was observed with respect to trouser size and the distal colon (largest size vs. smallest: HR $=2.56,95 \%$ CI: $1.55,4.24$; $P$-trend $<0.01$ ).

With respect to height, an inverse association was observed for rectosigmoid tumors $(5-\mathrm{cm}$ increase: $\mathrm{HR}=0.77,95 \% \mathrm{CI}$ : $0.65,0.93$; highest quintile vs. lowest: $\mathrm{HR}=0.38,95 \% \mathrm{CI}$ : $0.17,0.83(P$-trend $=0.01)$ ).

The strengths of the associations did not differ when data were split according to duration of follow-up ( $<8$ years vs. $\geq 8$ years). Tests for heterogeneity between tumor subtypes were not statistically significant for any risk factors considered.

\section{Associations with overall CRC in women}

In women, there were no statistically significant associations between BMI, BMI at age 20 years, BMI change, or trouser/skirt size and overall CRC risk (Table 4).

Height, modeled per 5-cm increase, was associated with a higher risk of total CRC $(\mathrm{HR}=1.09,95 \% \mathrm{CI}: 1.01,1.17)$. There was also an association when the highest quintile was 
Table 1. Baseline Characteristics of Male Subcohort Members ${ }^{a}$ According to Quintile of Body Mass Index, Netherlands Cohort Study, 1986

\begin{tabular}{|c|c|c|c|c|c|c|c|c|c|c|c|c|c|c|c|}
\hline & \multicolumn{15}{|c|}{ Quintile of $B M I^{b}$} \\
\hline & \multicolumn{3}{|c|}{1 (Lowest) $(n=289)$} & \multicolumn{3}{|c|}{$2(n=282)$} & \multicolumn{3}{|c|}{$3(n=280)$} & \multicolumn{3}{|c|}{$4(n=265)$} & \multicolumn{3}{|c|}{5 (Highest) $(n=249)$} \\
\hline & $\%$ & Mean (SD) & Median & $\%$ & Mean (SD) & Median & $\%$ & Mean (SD) & Median & $\%$ & Mean (SD) & Median & $\%$ & Mean (SD) & Median \\
\hline Age at baseline, years & & $61.0(4.3)$ & & & $61.0(4.1)$ & & & $60.9(4.2)$ & & & $61.0(4.2)$ & & & $61.2(4.2)$ & \\
\hline Total energy intake, kcal & & $2,115(445)$ & & & $2,194(485)$ & & & $2,202(480)$ & & & $2,147(505)$ & & & $2,172(523)$ & \\
\hline Weight, kg & & $67.9(6.1)$ & & & $74.2(5.6)$ & & & $77.4(5.4)$ & & & $81.4(6.3)$ & & & $88.9(8.9)$ & \\
\hline Height, cm & & $177.5(6.7)$ & & & $177.1(6.6)$ & & & $176.7(6.0)$ & & & $176.7(6.5)$ & & & $176.0(6.5)$ & \\
\hline Baseline BMI & & & 22.0 & & & 23.7 & & & 24.9 & & & 26.1 & & & 28.4 \\
\hline $\mathrm{BMl}$ at age 20 years & & & 18.9 & & & 20.5 & & & 21.7 & & & 22.9 & & & 24.7 \\
\hline Trouser size & & $50(2)$ & & & $50(2)$ & & & $52(2)$ & & & $52(2)$ & & & $54(2)$ & \\
\hline \multicolumn{16}{|l|}{$\begin{array}{l}\text { Occupational physical } \\
\text { activity, } \mathrm{kJ} / \text { minute }\end{array}$} \\
\hline$<8$ & 63 & & & 61 & & & 62 & & & 60 & & & 60 & & \\
\hline $8-<12$ & 26 & & & 25 & & & 24 & & & 26 & & & 26 & & \\
\hline$\geq 12$ & 11 & & & 13 & & & 14 & & & 14 & & & 14 & & \\
\hline \multicolumn{16}{|l|}{ Level of education } \\
\hline Primary school & 18 & & & 19 & & & 23 & & & 27 & & & 29 & & \\
\hline Junior/senior high school & 18 & & & 19 & & & 21 & & & 22 & & & 21 & & \\
\hline Higher vocational school & 39 & & & 40 & & & 36 & & & 33 & & & 35 & & \\
\hline University & 24 & & & 23 & & & 20 & & & 18 & & & 15 & & \\
\hline \multicolumn{16}{|l|}{ Smoking status } \\
\hline Never smoker & 12 & & & 11 & & & 11 & & & 12 & & & 14 & & \\
\hline Ex-smoker & 47 & & & 54 & & & 57 & & & 59 & & & 57 & & \\
\hline Current smoker & 41 & & & 35 & & & 32 & & & 30 & & & 29 & & \\
\hline \multicolumn{16}{|l|}{ Alcohol intake, g/day } \\
\hline 0 & 20 & & & 11 & & & 13 & & & 11 & & & 10 & & \\
\hline $0.1-4$ & 22 & & & 17 & & & 19 & & & 24 & & & 21 & & \\
\hline $5-14$ & 24 & & & 30 & & & 29 & & & 29 & & & 25 & & \\
\hline $15-29$ & 21 & & & 26 & & & 23 & & & 22 & & & 28 & & \\
\hline$\geq 30$ & 14 & & & 16 & & & 17 & & & 15 & & & 16 & & \\
\hline \multicolumn{16}{|l|}{$\begin{array}{l}\text { Family history of colorectal } \\
\text { cancer }\end{array}$} \\
\hline No & 96 & & & 95 & & & 93 & & & 96 & & & 95 & & \\
\hline Yes & 4 & & & 5 & & & 7 & & & 4 & & & 5 & & \\
\hline
\end{tabular}

a Subcohort members who were not missing data for the given exposure or any of the considered baseline characteristics.

${ }^{\mathrm{b}}$ Weight $(\mathrm{kg}) /$ height $(\mathrm{m})^{2}$. Range of BMI values: quintile 1, 16.1-23.0; quintile 2, 23.1-24.2; quintile 3, 24.3-25.3; quintile 4, 25.4-27.0; quintile 5, 27.1-39.6. 


\begin{tabular}{|c|c|c|c|c|c|c|c|c|c|c|c|c|c|c|c|}
\hline & \multicolumn{15}{|c|}{ Quintile of BMI $^{\mathrm{b}}$} \\
\hline & \multicolumn{3}{|c|}{1 (Lowest) $(n=359)$} & \multicolumn{3}{|c|}{$2(n=373)$} & \multicolumn{3}{|c|}{$3(n=369)$} & \multicolumn{3}{|c|}{$4(n=373)$} & \multicolumn{3}{|c|}{5 (Highest) $(n=358)$} \\
\hline & $\%$ & Mean (SD) & Median & $\%$ & Mean (SD) & Median & $\%$ & Mean (SD) & Median & $\%$ & Mean (SD) & Median & $\%$ & Mean (SD) & Median \\
\hline Age at baseline, years & & $61.2(4.5)$ & & & $61.0(4.3)$ & & & $61.1(4.2)$ & & & $6.16(4.2)$ & & & $61.4(4.1)$ & \\
\hline Total energy intake, kcal & & $1,719(412)$ & & & $1,710(401)$ & & & $1,725(420)$ & & & $1,655(350)$ & & & $1,630(372)$ & \\
\hline Weight, kg & & $57.4(5.6)$ & & & $63.6(4.6)$ & & & $67.8(4.9)$ & & & $71.5(5.6)$ & & & $81.7(8.5)$ & \\
\hline Height, $\mathrm{cm}$ & & $166.2(6.1)$ & & & $165.6(5.9)$ & & & $165.9(5.9)$ & & & $164.4(6.2)$ & & & $163.9(5.9)$ & \\
\hline Baseline BMI & & & 21.3 & & & 23.4 & & & 24.7 & & & 26.3 & & & 29.4 \\
\hline $\mathrm{BMI}$ at age 20 years & & & 18.0 & & & 20.0 & & & 21.3 & & & 22.6 & & & 24.6 \\
\hline Trouser/skirt size & & $40(2)$ & & & $42(2)$ & & & $43(2)$ & & & $44(2)$ & & & $46(2)$ & \\
\hline \multicolumn{16}{|l|}{$\begin{array}{l}\text { Recreational physical } \\
\text { activity, minutes/day }\end{array}$} \\
\hline$<30$ & 20 & & & 19 & & & 23 & & & 25 & & & 25 & & \\
\hline $30-<60$ & 30 & & & 32 & & & 32 & & & 33 & & & 33 & & \\
\hline $60-90$ & 25 & & & 26 & & & 23 & & & 21 & & & 21 & & \\
\hline$>90$ & 25 & & & 23 & & & 23 & & & 22 & & & 22 & & \\
\hline \multicolumn{16}{|l|}{ Level of education } \\
\hline Primary school & 26 & & & 27 & & & 31 & & & 38 & & & 39 & & \\
\hline Junior/senior high school & 17 & & & 24 & & & 26 & & & 25 & & & 28 & & \\
\hline Higher vocational school & 43 & & & 38 & & & 36 & & & 29 & & & 29 & & \\
\hline University & 13 & & & 12 & & & 7 & & & 8 & & & 4 & & \\
\hline \multicolumn{16}{|l|}{ Smoking status } \\
\hline Never smoker & 49 & & & 58 & & & 54 & & & 65 & & & 63 & & \\
\hline Ex-smoker & 24 & & & 18 & & & 25 & & & 21 & & & 19 & & \\
\hline Current smoker & 27 & & & 24 & & & 21 & & & 14 & & & 18 & & \\
\hline \multicolumn{16}{|l|}{ Alcohol intake, g/day } \\
\hline 0 & 28 & & & 31 & & & 32 & & & 33 & & & 37 & & \\
\hline $0.1-4$ & 33 & & & 35 & & & 37 & & & 39 & & & 37 & & \\
\hline $5-14$ & 22 & & & 20 & & & 18 & & & 18 & & & 15 & & \\
\hline $15-29$ & 12 & & & 11 & & & 9 & & & 8 & & & 8 & & \\
\hline$\geq 30$ & 6 & & & 3 & & & 4 & & & 3 & & & 3 & & \\
\hline \multicolumn{16}{|l|}{$\begin{array}{l}\text { Family history of colorectal } \\
\text { cancer }\end{array}$} \\
\hline No & 94 & & & 94 & & & 93 & & & 95 & & & 94 & & \\
\hline Yes & 6 & & & 6 & & & 7 & & & 5 & & & 6 & & \\
\hline
\end{tabular}

Abbreviations: BMI, body mass index; SD, standard deviation.

a Subcohort members who were not missing data for the given exposure or any of the considered baseline characteristics.

${ }^{\mathrm{b}}$ Weight $(\mathrm{kg}) /$ height $(\mathrm{m})^{2}$. Range of BMI values: quintile 1, 15.4-22.1; quintile 2, 22.2-23.8; quintile 3, 23.9-25.3; quintile 4, 25.4-27.5; quintile 5, 27.6-41.4. 
Table 3. Multivariate-Adjusted Hazard Ratios ${ }^{\text {a }}$ for the Association Between Anthropometric Variables and Colorectal Cancer in Men After 16.3 Years of Follow-up, by Anatomic Location, Netherlands Cohort Study, 1986-2002

\begin{tabular}{|c|c|c|c|c|c|c|c|c|c|c|c|c|c|c|c|c|}
\hline \multirow{3}{*}{ Exposure } & \multirow{3}{*}{$\begin{array}{l}\text { Person-Years } \\
\text { at Risk }\end{array}$} & \multicolumn{15}{|c|}{ Anatomic Location } \\
\hline & & \multicolumn{3}{|c|}{ Total Colorectal Cancer } & \multicolumn{3}{|c|}{ Proximal Colon } & \multicolumn{3}{|c|}{ Distal Colon } & \multicolumn{3}{|c|}{ Rectosigmoid Junction } & \multicolumn{3}{|c|}{ Rectum } \\
\hline & & $\begin{array}{l}\text { No. of } \\
\text { Cases }\end{array}$ & HR & $95 \% \mathrm{Cl}$ & $\begin{array}{l}\text { No. of } \\
\text { Cases }\end{array}$ & HR & $95 \% \mathrm{Cl}$ & $\begin{array}{l}\text { No. of } \\
\text { Cases }\end{array}$ & HR & $95 \% \mathrm{Cl}$ & $\begin{array}{l}\text { No. of } \\
\text { Cases }\end{array}$ & HR & $95 \% \mathrm{Cl}$ & $\begin{array}{l}\text { No. of } \\
\text { Cases }\end{array}$ & HR & $95 \% \mathrm{Cl}$ \\
\hline \multicolumn{17}{|l|}{$\mathrm{BMI}^{\mathrm{b}}$} \\
\hline Per 5-unit increase & 18,595 & 1,211 & 1.25 & $1.05,1.46$ & 327 & 1.19 & $0.92,1.54$ & 427 & 1.42 & $1.13,1.79$ & 125 & 1.49 & $1.05,2.11$ & 299 & 1.02 & $0.79,1.32$ \\
\hline Q1 & 3,772 & 232 & 1 & & 61 & 1 & & 78 & 1 & & 16 & 1 & & 60 & 1 & \\
\hline Q2 & 3,931 & 238 & 0.95 & $0.74,1.24$ & 75 & 1.15 & $0.78,1.70$ & 78 & 0.92 & $0.64,1.34$ & 24 & 1.34 & $0.67,2.61$ & 59 & 0.92 & $0.61,1.39$ \\
\hline Q3 & 3,889 & 240 & 0.99 & $0.77,1.28$ & 58 & 0.89 & $0.59,1.33$ & 89 & 1.07 & $0.75,1.53$ & 24 & 1.34 & $0.69,2.60$ & 59 & 0.90 & $0.60,1.35$ \\
\hline Q4 & 3,688 & 247 & 1.05 & $0.81,1.36$ & 59 & 0.93 & $0.62,1.40$ & 89 & 1.10 & $0.77,1.58$ & 34 & 2.04 & $1.08,3.82$ & 60 & 0.93 & $0.62,1.41$ \\
\hline Q5 & 3,315 & 254 & 1.25 & $0.96,1.62$ & 74 & 1.35 & $0.90,1.98$ & 93 & 1.38 & $0.95,1.98$ & 27 & 1.62 & $0.85,3.10$ & 61 & 1.01 & $0.67,1.51$ \\
\hline$P$-trend & & & 0.08 & & & 0.43 & & & 0.05 & & & 0.05 & & & 0.96 & \\
\hline \multicolumn{17}{|l|}{$\mathrm{BMl}$ at age 20 years $^{\mathrm{C}}$} \\
\hline Per 5-unit increase & 18,595 & 1,211 & 1.15 & $0.97,1.37$ & 327 & 0.98 & $0.75,1.27$ & 427 & 1.09 & $1.00,1.19$ & 125 & 1.40 & $0.98,2.01$ & 299 & 1.12 & $0.86,1.46$ \\
\hline Q1 & 3,771 & 238 & 1 & & 69 & 1 & & 84 & 1 & & 21 & 1 & & 50 & 1 & \\
\hline Q2 & 4,090 & 240 & 0.92 & $0.72,1.19$ & 69 & 0.98 & $0.66,1.44$ & 82 & 0.96 & $0.67,1.37$ & 19 & 0.85 & $0.45,1.63$ & 62 & 1.18 & $0.77,1.80$ \\
\hline Q3 & 3,723 & 229 & 0.99 & $0.77,1.28$ & 62 & 0.93 & $0.63,1.38$ & 72 & 0.84 & $0.58,1.22$ & 26 & 1.24 & $0.68,2.26$ & 59 & 1.16 & $0.75,1.79$ \\
\hline Q4 & 3,622 & 254 & 1.09 & $0.84,1.41$ & 60 & 0.86 & $0.58,1.29$ & 89 & 1.03 & $0.72,1.47$ & 31 & 1.43 & $0.79,2.60$ & 69 & 1.34 & $0.88,2.03$ \\
\hline Q5 & 3,389 & 250 & 1.21 & $0.93,1.56$ & 67 & 1.05 & $0.72,1.56$ & 100 & 1.47 & $1.03,2.08$ & 28 & 1.37 & $0.75,2.51$ & 59 & 1.11 & $0.73,1.71$ \\
\hline$P$-trend & & & 0.07 & & & 0.97 & & & 0.05 & & & 0.10 & & & 0.46 & \\
\hline \multicolumn{17}{|l|}{ BMI change $^{d}$} \\
\hline$<0$ & 1,646 & 103 & 0.87 & $0.63,1.19$ & 27 & 0.91 & $0.56,1.47$ & 36 & 0.87 & $0.56,1.36$ & 7 & 0.41 & $0.17,1.02$ & 29 & 0.99 & $0.61,1.61$ \\
\hline $0-<4$ & 10,298 & 657 & 1 & & 178 & 1 & & 224 & 1 & & 74 & 1 & & 163 & 1 & \\
\hline $4-<8$ & 5,701 & 375 & 1.07 & $0.88,1.30$ & 96 & 0.97 & $0.71,1.32$ & 140 & 1.23 & $0.93,1.62$ & 37 & 1.01 & $0.63,1.61$ & 91 & 1.02 & $0.75,1.40$ \\
\hline$\geq 8$ & 950 & 76 & 1.38 & $0.93,2.05$ & 26 & 1.59 & $0.93,2.72$ & 27 & 1.56 & $0.91,2.65$ & 7 & 1.29 & $0.51,3.26$ & 16 & 1.16 & $0.63,2.15$ \\
\hline$P$-trend & & & 0.09 & & & 0.33 & & & 0.05 & & & 0.19 & & & 0.73 & \\
\hline \multicolumn{17}{|l|}{ Height, $\mathrm{cm}^{\mathrm{e}}$} \\
\hline Per 5-cm increase & 18,595 & 1,211 & 0.96 & $0.89,1.04$ & 327 & 1.01 & $0.90,1.13$ & 427 & 0.99 & $0.89,1.11$ & 125 & 0.77 & $0.65,0.93$ & 299 & 0.95 & $0.85,1.07$ \\
\hline Q1 & 5,047 & 337 & 1 & & 94 & 1 & & 96 & 1 & & 32 & 1 & & 72 & 1 & \\
\hline Q2 & 2,781 & 167 & 0.85 & $0.65,1.12$ & 39 & 0.73 & $0.48,1.12$ & 66 & 1.13 & $0.77,1.65$ & 26 & 0.73 & $0.41,1.29$ & 62 & 0.84 & $0.56,1.26$ \\
\hline Q3 & 3,819 & 262 & 0.94 & $0.73,1.20$ & 68 & 0.91 & $0.62,1.31$ & 91 & 1.03 & $0.73,1.47$ & 34 & 0.90 & $0.51,1.57$ & 63 & 0.91 & $0.60,1.37$ \\
\hline Q4 & 3,480 & 224 & 0.84 & $0.64,1.09$ & 59 & 0.82 & $0.55,1.22$ & 84 & 0.96 & $0.65,1.41$ & 19 & 0.54 & $0.27,1.06$ & 58 & 0.92 & $0.60,1.42$ \\
\hline Q5 & 3,469 & 221 & 0.80 & $0.60,1.08$ & 67 & 0.90 & $0.59,1.37$ & 90 & 0.97 & $0.63,1.48$ & 14 & 0.38 & $0.17,0.83$ & 44 & 0.71 & $0.43,1.17$ \\
\hline$P$-trend & & & 0.16 & & & 0.66 & & & 0.70 & & & 0.01 & & & 0.35 & \\
\hline
\end{tabular}




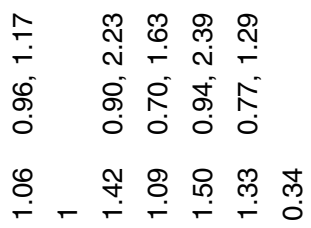

尺

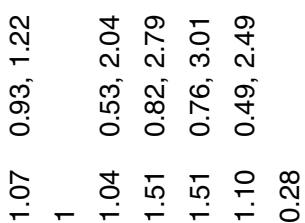

ำㄴ 누

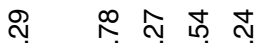

$\sim$ त ले

ণั

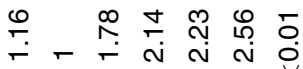

ฟิ กั

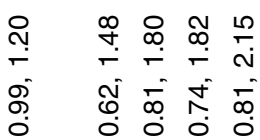

우 _

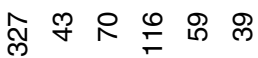

으 항 으 옥

$\dot{2}+\frac{1}{4} \sin$

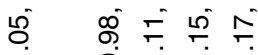

- $0 \div-5$

$\stackrel{ }{\leftarrow}$

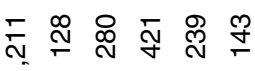

ᄂூด \& 品

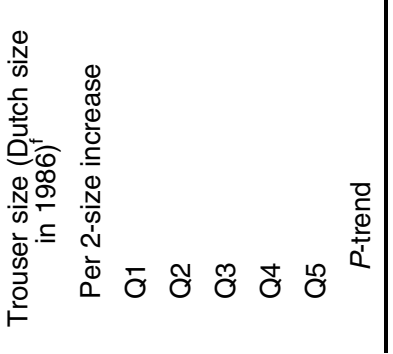

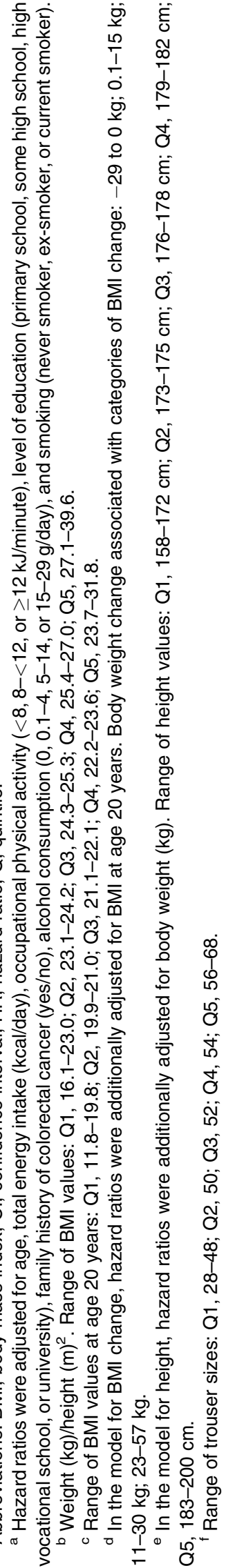

compared with the lowest quintile (HR $=1.32,95 \%$ CI: $1.03,1.71 ; P$-trend $=0.05)$.

\section{Associations by tumor subsite in women}

Associations by subsite in women were not as clear as those in men. With respect to body fat, women with the largest trouser/skirt size had a borderline statistically significant risk of proximal colon tumors compared with those with the smallest size $(\mathrm{HR}=1.46,95 \%$ CI: $0.98,2.18)$, but there was no clear trend $(P$-trend $=0.46)$.

There was a borderline statistically significant association between height and distal colon tumors (HR $=1.11,95 \%$ CI: $0.99,1.24)$ and rectal tumors (HR $=1.14,95 \%$ CI: 0.98 , 1.32 ) when data were modeled per $5-\mathrm{cm}$ increase. Women in the highest quintile also had a statistically significant risk of distal tumors when compared with those in the lowest quintile $(\mathrm{HR}=1.53,95 \% \mathrm{CI}: 1.03,2.27 ; P$-trend $=0.05)$. This association was borderline statistically significant for rectal tumors $(\mathrm{HR}=1.49,95 \% \mathrm{CI}$ : 0.98, 2.27; $P$-trend $=0.06)$.

When data were split at 8 years of follow-up, it appeared that associations for all anthropometric variables were attenuated over time (data not shown). Tests for heterogeneity between tumor subtypes were not statistically significant for any risk factors considered.

\section{Interaction between trouser/skirt size and physical activity}

Table 5 shows hazard ratios for the interaction between trouser size and occupational physical activity in men. Compared with the reference category of men with a small trouser size and a high level of physical activity, there were no statistically significant associations for total CRC, but men with a small trouser size and a low level of physical activity were at increased risk of distal colon tumors $(\mathrm{HR}=1.63$, $95 \%$ CI: 1.03, 2.56). A borderline statistically significant association was also observed for men with a large trouser size and all levels of physical activity with respect to tumors of the distal colon.

Compared with the reference category of women with a small trouser/skirt size and a high level of physical activity (Table 6), the greatest risk for each subtype considered appeared to be for persons with the highest trouser/skirt size and the lowest level of physical activity (for total CRC, $\mathrm{HR}=1.83$, $95 \%$ CI: 1.28, 2.63; for proximal colon tumors, $\mathrm{HR}=1.70$, $95 \%$ CI: $1.08,2.67$; for distal colon tumors, $\mathrm{HR}=1.95,95 \%$ CI: $1.21,3.17$; for rectal tumors, $\mathrm{HR}=2.56,95 \% \mathrm{CI}: 1.36$, 4.79). The interaction between trouser/skirt size and physical activity was statistically significant only for the proximal colon $(P<0.05)$.

\section{DISCUSSION}

We observed that in men, body fat, as indicated by BMI, BMI at age 20 years, BMI change, and trouser size, appeared to be more associated with distal tumors than with tumors at other anatomic subsites; however, associations with respect to height were less clear. Body size appeared to be unrelated to CRC outcomes in women, except for height, which was 
Table 4. Multivariate-Adjusted Hazard Ratios ${ }^{a}$ for the Association Between Anthropometric Variables and Colorectal Cancer Endpoints in Women After 16.3 Years of Follow-up, by Anatomic Location, Netherlands Cohort Study, 1986-2002

\begin{tabular}{|c|c|c|c|c|c|c|c|c|c|c|c|c|c|c|c|c|}
\hline \multirow{3}{*}{ Exposure } & \multirow{3}{*}{$\begin{array}{c}\text { Person-Years } \\
\text { at Risk }\end{array}$} & \multicolumn{15}{|c|}{ Anatomic Location } \\
\hline & & \multicolumn{3}{|c|}{ Total Colorectal Cancer } & \multicolumn{3}{|c|}{ Proximal Colon } & \multicolumn{3}{|c|}{ Distal Colon } & \multicolumn{3}{|c|}{ Rectosigmoid Junction } & \multicolumn{3}{|c|}{ Rectum } \\
\hline & & $\begin{array}{l}\text { No. of } \\
\text { Cases }\end{array}$ & HR & $95 \% \mathrm{Cl}$ & $\begin{array}{l}\text { No. of } \\
\text { Cases }\end{array}$ & HR & $95 \% \mathrm{Cl}$ & $\begin{array}{l}\text { No. of } \\
\text { Cases }\end{array}$ & HR & $95 \% \mathrm{Cl}$ & $\begin{array}{l}\text { No. of } \\
\text { Cases }\end{array}$ & HR & $95 \% \mathrm{Cl}$ & $\begin{array}{l}\text { No. of } \\
\text { Cases }\end{array}$ & HR & $95 \% \mathrm{Cl}$ \\
\hline \multicolumn{17}{|l|}{$\mathrm{BMI}^{\mathrm{b}}$} \\
\hline Per 5-unit increase & 27,195 & 1,106 & 0.98 & $0.88,1.10$ & 459 & 1.02 & $0.87,1.18$ & 327 & 0.95 & $0.79,1.14$ & 87 & 1.01 & $0.74,1.38$ & 205 & 1.05 & $0.83,1.31$ \\
\hline Q1 & 5,238 & 228 & 1 & & 94 & 1 & & 70 & 1 & & 16 & 1 & & 42 & 1 & \\
\hline Q2 & 5,623 & 211 & 0.88 & $0.69,1.13$ & 87 & 0.88 & $0.63,1.23$ & 62 & 0.85 & $0.58,1.24$ & 17 & 1.08 & $0.53,2.21$ & 41 & 1.00 & $0.63,1.58$ \\
\hline Q3 & 5,499 & 223 & 0.94 & $0.73,1.20$ & 94 & 0.99 & $0.71,1.40$ & 58 & 0.81 & $0.55,1.19$ & 26 & 1.86 & $0.94,3.69$ & 38 & 0.87 & $0.54,1.40$ \\
\hline Q4 & 5,606 & 222 & 0.91 & $0.71,1.16$ & 95 & 0.98 & $0.70,1.37$ & 64 & 0.85 & $0.58,1.23$ & 13 & 0.85 & $0.40,1.84$ & 40 & 0.93 & $0.58,1.49$ \\
\hline Q5 & 5,229 & 222 & 0.97 & $0.76,1.24$ & 89 & 0.91 & $0.65,1.28$ & 73 & 1.04 & $0.72,1.50$ & 15 & 1.06 & $0.50,2.26$ & 44 & 1.07 & $0.67,1.60$ \\
\hline$P$-trend & & & 0.90 & & & 0.84 & & & 0.84 & & & 0.93 & & & 0.90 & \\
\hline \multicolumn{17}{|l|}{$\mathrm{BMI}$ at age 20 years $^{\mathrm{c}}$} \\
\hline Per 5-unit increase & 27,195 & 1,106 & 1.04 & $0.90,1.19$ & 459 & 1.03 & $0.84,1.25$ & 327 & 1.05 & $0.85,1.31$ & 87 & 0.91 & $0.61,1.35$ & 205 & 1.06 & $0.82,1.36$ \\
\hline Q1 & 5,628 & 213 & 1 & & 90 & 1 & & 60 & 1 & & 19 & 1 & & 38 & 1 & \\
\hline Q2 & 5,247 & 227 & 1.21 & $0.95,1.56$ & 100 & 1.28 & $0.90,1.76$ & 67 & 1.22 & $0.83,1.79$ & 18 & 1.09 & $0.56,1.13$ & 43 & 1.26 & $0.78,2.01$ \\
\hline Q3 & 5,212 & 236 & 1.25 & $0.98,1.61$ & 96 & 1.15 & $0.82,1.60$ & 74 & 1.36 & $0.95,1.99$ & 18 & 0.97 & $0.50,1.89$ & 46 & 1.34 & $0.84,2.13$ \\
\hline Q4 & 5,576 & 212 & 1.08 & $0.84,1.38$ & 87 & 0.98 & $0.70,1.38$ & 65 & 1.21 & $0.82,1.79$ & 15 & 0.85 & $0.42,1.72$ & 35 & 0.98 & $0.60,1.61$ \\
\hline Q5 & 5,532 & 218 & 1.12 & $0.87,1.43$ & 86 & 1.14 & $0.81,1.62$ & 61 & 1.09 & $0.74,1.62$ & 17 & 1.07 & $0.54,2.12$ & 43 & 1.29 & $0.80,2.07$ \\
\hline$P$-trend & & & 0.68 & & & 0.97 & & & 0.68 & & & 0.89 & & & 0.62 & \\
\hline \multicolumn{17}{|l|}{ BMI change $^{d}$} \\
\hline$<0$ & 3,268 & 134 & 0.94 & $0.73,1.22$ & 51 & 0.86 & $0.60,1.23$ & 44 & 1.08 & $0.72,1.60$ & 12 & 1.26 & $0.63,2.50$ & 19 & 0.67 & $0.39,1.14$ \\
\hline $0-<4$ & 12,177 & 507 & 1 & & 212 & 1 & & 147 & 1 & & 37 & 1 & & 97 & 1 & \\
\hline $4-<8$ & 8,787 & 343 & 0.89 & $0.74,1.06$ & 144 & 0.86 & $0.67,1.10$ & 98 & 0.89 & $0.67,1.20$ & 26 & 0.97 & $0.56,1.68$ & 69 & 0.96 & $0.68,1.35$ \\
\hline$\geq 8$ & 2,963 & 122 & 0.95 & $0.73,1.24$ & 52 & 0.98 & $0.68,1.40$ & 38 & 1.00 & $0.67,1.50$ & 12 & 1.40 & $0.69,2.82$ & 20 & 0.83 & $0.49,1.39$ \\
\hline$P$-trend & & & 0.55 & & & 0.86 & & & 0.58 & & & 0.83 & & & 0.78 & \\
\hline \multicolumn{17}{|l|}{ Height, $\mathrm{cm}^{\mathrm{e}}$} \\
\hline Per $5 \mathrm{~cm}$ & 27,195 & 1,106 & 1.09 & $1.01,1.17$ & 459 & 1.04 & $0.95,1.15$ & 327 & 1.11 & $0.99,1.24$ & 87 & 1.02 & $0.82,1.25$ & 205 & 1.14 & $0.98,1.32$ \\
\hline Q1 & 6,353 & 250 & 1 & & 104 & 1 & & 68 & 1 & & 24 & 1 & & 49 & 1 & \\
\hline Q2 & 4,932 & 186 & 0.96 & $0.75,1.23$ & 89 & 1.11 & $0.80,1.54$ & 62 & 1.17 & $0.80,1.72$ & 10 & 0.53 & $0.25,1.12$ & 20 & 0.52 & $0.30,0.89$ \\
\hline Q3 & 5,599 & 209 & 0.97 & $0.77,1.24$ & 103 & 1.15 & $0.83,1.58$ & 49 & 0.84 & $0.56,1.25$ & 13 & 0.61 & $0.30,1.24$ & 40 & 0.93 & $0.60,1.45$ \\
\hline Q4 & 5,708 & 231 & 1.03 & $0.81,1.31$ & 78 & 0.83 & $0.59,1.18$ & 76 & 1.28 & $0.88,1.86$ & 23 & 1.02 & $0.55,1.86$ & 45 & 1.04 & $0.68,1.59$ \\
\hline Q5 & 4,603 & 230 & 1.32 & $1.03,1.71$ & 85 & 1.19 & $0.84,1.70$ & 72 & 1.53 & $1.03,2.27$ & 17 & 0.90 & $0.44,1.84$ & 51 & 1.49 & $0.98,2.27$ \\
\hline$P$-trend & & & 0.05 & & & 0.67 & & & 0.05 & & & 0.78 & & & 0.06 & \\
\hline
\end{tabular}




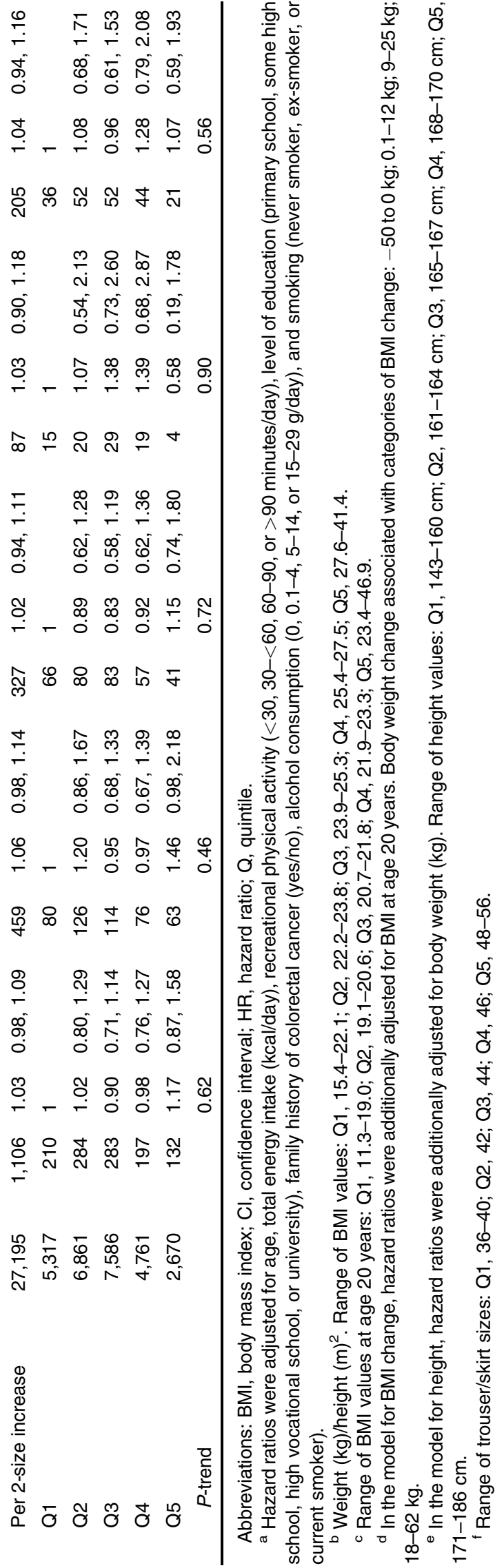

related positively to cancers of the distal colon and rectum. In general, our observations, especially in men, highlight the importance of examining CRC risks according to tumor subsite, because stronger associations were observed for individual subsites than with overall risk.

Strengths of this study include the prospective cohort design and the large population, in combination with sufficient follow-up time to allow precise analysis of CRC risk at different subsites. The NLCS has almost complete ascertainment of CRC cases, and follow-up of the subcohort is almost $100 \%$. Although measures of body size in this study were obtained by self-report, there are many examples in the literature showing that this method is a reasonably valid and reliable tool for assessing body weight and height in cohort studies (33-36). A weakness of this study was the small number of rectosigmoid and rectal tumors and the subsequently limited statistical power.

Investigators in several case-control studies have considered the association between BMI and site-specific tumors of the colon in men, and they have generally reported a stronger risk for tumors of the distal colon than for tumors of the proximal colon (6-10). This is supported by the results of a recent prospective cohort study carried out by Laake et al. (11). In our study, we considered several indicators of body fat in addition to BMI, including BMI at age 20 years, BMI change, and trouser/skirt size as a proxy for waist circumference, and we consistently observed an elevated risk of distal colon tumors as compared with tumors at other subsites. Although we did not observe statistically significant heterogeneity between proximal and distal colon tumors as Laake et al. (11) did, the bootstrapping method we used is quite conservative. The weaker associations that we observed with respect to associations between body fat and the rectum align with previous research (1), but we also observed a positive association between BMI and tumors of the rectosigmoid junction. To our knowledge, no other prospective study has considered the association between anthropometric measures and the rectosigmoid junction. This association is plausible, because the rectosigmoid junction and distal colon are anatomically linked. Our findings should be replicated in other prospective studies, but they support the hypothesis that the etiology of CRC tumors differs between anatomic subsites of the colorectal tract in men.

We did not observe clear associations between BMI, BMI at age 20 years, or BMI change and CRC risk in women, but our results suggest that women with a high trouser/skirt size are at increased risk of proximal colon tumors, although this association did not reach statistical significance. In contrast, positive associations between BMI and distal colon tumors have been reported in prospective cohort studies of Swedish (12) and Norwegian (11) women. In an American cohort, both BMI and waist circumference were associated with proximal and distal tumors (13). The proportion of obese women in the NLCS is small, and perhaps this prevented us from detecting associations of similar strength. It has also been suggested that the impact of a given risk factor along the length of the large bowel may differ according to the prevalence of other environmental factors and, thus, according to sex and country (2).

Alternatively, it is plausible that a metabolic profile reflecting a combination of risk factors has a greater influence 
Table 5. Multivariate-Adjusted Hazard Ratios ${ }^{a}$ for the Interaction Between Occupational Physical Activity and Trouser Size in the Risk of Colorectal Cancer Among Men, Netherlands Cohort Study, 1986-2002

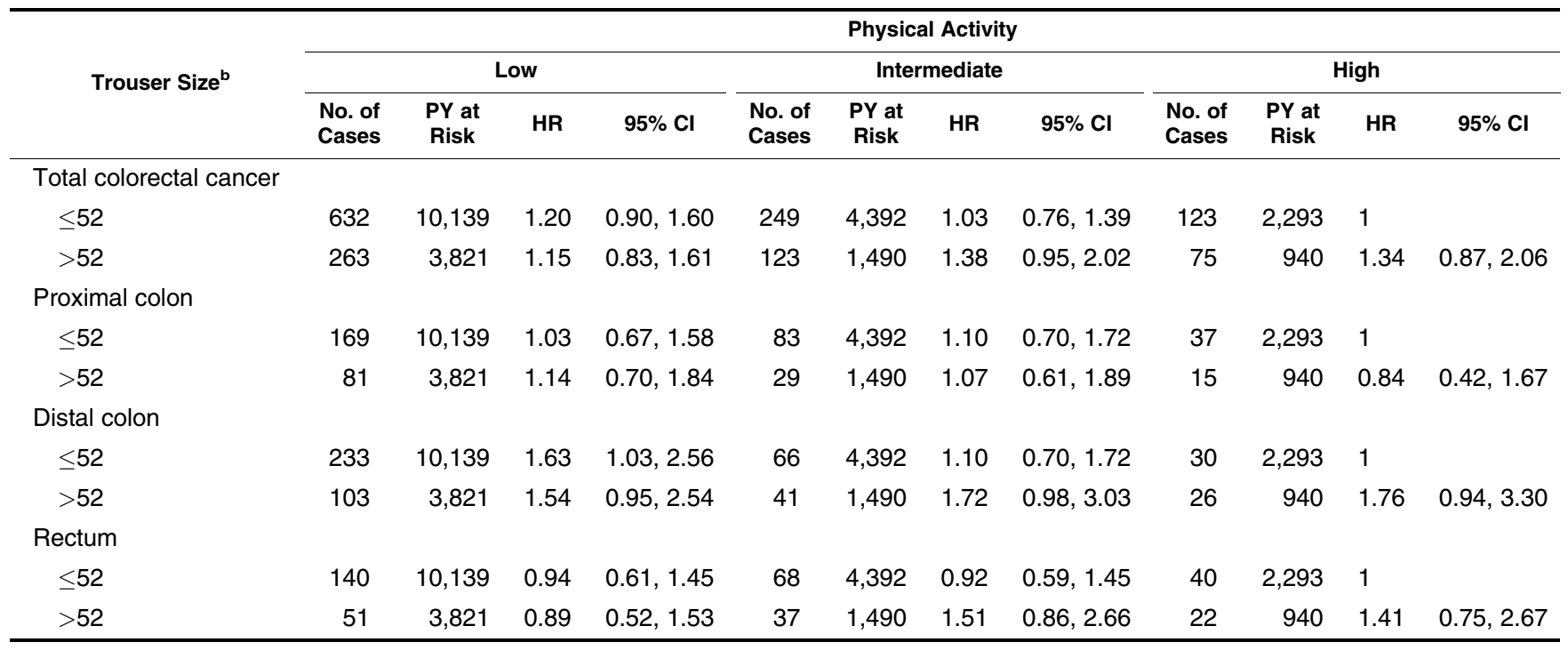

Abbreviations: $\mathrm{Cl}$, confidence interval; $\mathrm{HR}$, hazard ratio; PY, person-years.

${ }^{a}$ Hazard ratios were adjusted for trouser size, physical activity, total energy intake, family history of colorectal cancer, socioeconomic status, alcohol consumption, and smoking status.

${ }^{\mathrm{b}}$ Dutch size in 1986.

on cancer risk than do the individual effects of body size and obesity $(37,38)$. Because evidence suggests that fat distribution is more important than body weight or BMI for CRC risk, especially in women (14), we investigated the interaction with physical activity using trouser/skirt size. Our observations for women were intriguing. As noted above, we did not observe statistically significant associations with respect to trouser/skirt size in our general analysis. However, when we considered the interaction between trouser/skirt size and physical activity, it appeared that women with a large trouser/skirt size and the lowest level of physical activity were at the greatest risk of CRC compared with women with a small trouser/skirt

Table 6. Multivariate-Adjusted Hazard Ratios ${ }^{a}$ for the Interaction Between Nonoccupational Physical Activity and Trouser/Skirt Size in the Risk of Colorectal Cancer Among Women, Netherlands Cohort Study, 1986-2002

\begin{tabular}{|c|c|c|c|c|c|c|c|c|c|c|c|c|}
\hline \multirow{3}{*}{ Trouser/Skirt Size $^{b}$} & \multicolumn{12}{|c|}{ Physical Activity } \\
\hline & \multicolumn{4}{|c|}{ Low } & \multicolumn{4}{|c|}{ Intermediate } & \multicolumn{4}{|c|}{ High } \\
\hline & $\begin{array}{l}\text { No. of } \\
\text { Cases }\end{array}$ & $\begin{array}{l}\text { PY at } \\
\text { Risk }\end{array}$ & HR & $95 \% \mathrm{Cl}$ & $\begin{array}{l}\text { No. of } \\
\text { Cases }\end{array}$ & $\begin{array}{l}\text { PY at } \\
\text { Risk }\end{array}$ & HR & $95 \% \mathrm{Cl}$ & $\begin{array}{l}\text { No. of } \\
\text { Cases }\end{array}$ & $\begin{array}{l}\text { PY at } \\
\text { Risk }\end{array}$ & HR & $95 \% \mathrm{Cl}$ \\
\hline \multicolumn{13}{|l|}{ Total colorectal cancer } \\
\hline$\leq 44$ & 159 & 4,471 & 1.45 & $1.08,1.94$ & 357 & 12,026 & 1.25 & $0.97,1.60$ & 122 & 5,054 & 1 & \\
\hline$>44$ & 97 & 2,283 & 1.83 & $1.28,2.63$ & 116 & 4,447 & 1.14 & $0.82,1.59$ & 51 & 1,617 & 1.44 & $0.95,2.18$ \\
\hline$\leq 44$ & 89 & 4,471 & 1.60 & $1.10,2.31$ & 203 & 12,026 & 1.40 & $1.02,1.93$ & 63 & 5,054 & 1 & \\
\hline$>44$ & 52 & 2,283 & 1.70 & $1.08,2.67$ & 66 & 4,447 & 1.16 & $0.76,1.77$ & 33 & 1,617 & 1.69 & $1.03,2.79$ \\
\hline \multicolumn{13}{|l|}{ Distal colon } \\
\hline$\leq 44$ & 63 & 4,471 & 1.28 & $0.86,1.91$ & 141 & 12,026 & 1.08 & $0.77,1.52$ & 54 & 5,054 & 1 & \\
\hline$>44$ & 42 & 2,283 & 1.95 & $1.21,3.17$ & 50 & 4,447 & 1.18 & $0.74,1.84$ & 17 & 1,617 & 1.12 & $0.61,2.03$ \\
\hline
\end{tabular}

Abbreviations: $\mathrm{Cl}$, confidence interval; $\mathrm{HR}$, hazard ratio; $\mathrm{PY}$, person-years.

* $P$ for interaction $<0.05$.

${ }^{a}$ Hazard ratios were adjusted for trouser/skirt size, physical activity, total energy intake, family history of colorectal cancer, socioeconomic status, alcohol consumption, and smoking status.

${ }^{\mathrm{b}}$ Dutch size in 1986. 
size and a high level of physical activity. This risk was highest for rectal tumors; however, the interaction was statistically significant only for tumors of the proximal colon. These findings require confirmation in other prospective studies, but they support case-control data $(9,39)$. They also highlight that underlying population levels of physical activity can impair or enhance the ability to identify CRC associations with other risk factors (31), such as body fat.

Height is a reflection of earlier life exposures, such as childhood energy intake (40). In our study, height was associated with increased risk of CRC in women, which is in accordance with previous research $(1,13,14,41,42)$. In a recent report, Oxentenko et al. (13) suggested that risk is greater for tumors of the proximal colon, whereas our data suggest that risk is greater for tumors of the distal colon. However, in the former study, Oxentenko et al. did not report conducting tests for heterogeneity, and in our study, such tests did not reach statistical significance. We did not observe any association with respect to height and CRC risk in men, and we actually observed an inverse association for tumors of the rectosigmoid junction. We cannot explain this observation, and we suggest that it may be a chance finding. We have shown previously in the NLCS population that energy restriction during childhood is inversely related to later CRC risk (43); therefore, while we have not observed strong associations between height and CRC in the present study, it is plausible that early-life nutritional factors may influence different carcinogenic pathways than later-life exposure to increased body fat (13).

We can only speculate as to why body size and body fat in particular might differentially influence the etiology of colorectal tumors at different subsites. Obesity is associated with a chronic state of low-grade inflammation and thus increased circulatory levels of inflammatory markers like C-reactive protein, interleukin-6, and tumor necrosis factor $\alpha$ (44). These in turn may induce insulin resistance and hyperinsulinemia. It is hypothesized that such conditions influence CRC risk (45-47); however, it remains unclear how they directly or indirectly influence specific tumor subsites. There are some distinct differences between the proximal colon and the distal colon, both anatomically and genetically (3-5). The distal colon is associated more with physical/chemical stimuli and associated less with water absorption and electrolyte transport than the proximal colon (3). It has been shown that tumors characterized by chromosomal instability are more associated with tumors of the distal colon, whereas epigenetic changes like methylation are more associated with tumors of the proximal colon (48). Interestingly, recent studies have suggested that overweight and obesity do not appear to differentially influence CRC risk via epigenetic mechanisms and microsatellite instability $(20,22,49)$. Based on the observation that body fat appears to be associated with a higher risk of distal colon tumors, a plausible hypothesis is that obesity and its associated process may influence risk via the chromosomal instability pathway. However, to our knowledge, this has not been investigated in any population-based studies. It is clear that the association between metabolic and hormonal risk factors and CRC is complex, and more research in this area is needed to elucidate clear mechanisms and how these mechanisms might differ with respect to tumor subsite.
In this Dutch population, body fat—especially abdominal fat, as indicated by trouser size-was associated with tumors of the distal colon in men. In women, the influence of body fat on CRC risk may differ according to level of physical activity. Height was associated with CRC risk only in women. More research is needed to fully elucidate how body size may influence subsite-specific pathways of CRC carcinogenesis.

\section{ACKNOWLEDGMENTS}

Author affiliations: Department of Epidemiology, GROW School for Oncology and Developmental Biology, Maastricht University, Maastricht, the Netherlands (Laura A. E. Hughes, Colinda C. J. M. Simons, Piet A. van den Brandt, Matty P. Weijenberg); Department of Pathology, GROW School for Oncology and Developmental Biology, Maastricht University, Maastricht, the Netherlands (Manon van Engeland); and Department of Prevention and Health, TNO Quality of Life, Leiden, the Netherlands (R. Alexandra Goldbohm).

This study was supported by a grant from the World Cancer Research Fund (grant 2007/54) to Dr. Matty P. Weijenberg.

The authors thank the staffs of the municipal cancer registries (IKA, IKL, IKMN, IKN, IKO, IKR, IKST, IKW, IKZ, and VIKC) and the Netherlands nationwide pathology registry (PALGA). They also thank S. van de Crommert and $\mathrm{J}$. Nelissen for their assistance with data entry and data management; A. Volovics and A. Kester for statistical advice; Dr. L. Schouten, C. de Zwart, M. Moll, W. van Dijk, M. Jansen, and A. Pisters for data management assistance; and $\mathrm{H}$. van Montfort, T. van Moergastel, L. van den Bosch, and R. Schmeitz for programming assistance.

Conflict of interest: none declared.

\section{REFERENCES}

1. World Cancer Research Fund/American Institute for Cancer Research. Food, Nutrition, Physical Activity, and the Prevention of Cancer: A Global Perspective. Washington, DC: American Institute for Cancer Research; 2007.

2. Morois S, Mesrine S, Josset M, et al. Anthropometric factors in adulthood and risk of colorectal adenomas: the French E3NEPIC prospective cohort. Am J Epidemiol. 2010;172(10): 1166-1180.

3. Li FY, Lai MD. Colorectal cancer, one entity or three. J Zhejiang Univ Sci B. 2009;10(3):219-229.

4. Breivik J, Lothe RA, Meling GI, et al. Different genetic pathways to proximal and distal colorectal cancer influenced by sex-related factors. Int J Cancer. 1997;74(6):664-669.

5. Lindblom A. Different mechanisms in the tumorigenesis of proximal and distal colon cancers. Curr Opin Oncol. 2001; 13(1):63-69.

6. Caan BJ, Coates AO, Slattery ML, et al. Body size and the risk of colon cancer in a large case-control study. Int J Obes Relat Metab Disord. 1998;22(2):178-184.

7. Gerhardsson de Verdier M, Hagman U, Steineck G, et al. Diet, body mass and colorectal cancer: a case-referent study in Stockholm. Int J Cancer. 1990;46(5):832-838. 
8. Russo A, Franceschi S, La Vecchia C, et al. Body size and colorectal-cancer risk. Int J Cancer. 1998;78(2):161-165.

9. Slattery ML, Potter J, Caan B, et al. Energy balance and colon cancer-beyond physical activity. Cancer Res. 1997;57(1):75-80.

10. West DW, Slattery ML, Robison LM, et al. Dietary intake and colon cancer: sex- and anatomic site-specific associations. Am J Epidemiol. 1989;130(5):883-894.

11. Laake I, Thune I, Selmer R, et al. A prospective study of body mass index, weight change, and risk of cancer in the proximal and distal colon. Cancer Epidemiol Biomarkers Prev. 2010; 19(6):1511-1522.

12. Terry P, Giovannucci E, Bergkvist L, et al. Body weight and colorectal cancer risk in a cohort of Swedish women: relation varies by age and cancer site. Br J Cancer. 2001;85(3):346-349.

13. Oxentenko AS, Bardia A, Vierkant RA, et al. Body size and incident colorectal cancer: a prospective study of older women. Cancer Prev Res (Phila). 2010;3(12):1608-1620.

14. Pischon T, Lahmann PH, Boeing $\mathrm{H}$, et al. Body size and risk of colon and rectal cancer in the European Prospective Investigation Into Cancer and Nutrition (EPIC). J Natl Cancer Inst. 2006;98(13):920-931.

15. Pischon T, Nöthlings U, Boeing H. Obesity and cancer. Proc Nutr Soc. 2008;67(2):128-145.

16. Derks S, Postma C, Carvalho B, et al. Integrated analysis of chromosomal, microsatellite and epigenetic instability in colorectal cancer identifies specific associations between promoter methylation of pivotal tumour suppressor and DNA repair genes and specific chromosomal alterations. Carcinogenesis. 2008;29(2):434-439.

17. Barault L, Charon-Barra C, Jooste V, et al. Hypermethylator phenotype in sporadic colon cancer: study on a populationbased series of 582 cases. Cancer Res. 2008;68(20):8541-8546.

18. Samowitz WS, Albertsen H, Herrick J, et al. Evaluation of a large, population-based sample supports a $\mathrm{CpG}$ island methylator phenotype in colon cancer. Gastroenterology. 2005; 129(3):837-845.

19. Weisenberger DJ, Siegmund KD, Campan M, et al. CpG island methylator phenotype underlies sporadic microsatellite instability and is tightly associated with BRAF mutation in colorectal cancer. Nat Genet. 2006;38(7):787-793.

20. Campbell PT, Jacobs ET, Ulrich CM, et al. Case-control study of overweight, obesity, and colorectal cancer risk, overall and by tumor microsatellite instability status. Colon Cancer Family Registry. J Natl Cancer Inst. 2010;102(6):391-400.

21. Hughes LA, Simons CC, van den Brandt PA, et al. Body size, physical activity and risk of colorectal cancer with or without the CpG Island Methylator Phenotype (CIMP). PloS One. 2011;6(4):e18571. (doi:10.1371/journal.pone.0018571).

22. Slattery ML, Curtin K, Sweeney C, et al. Diet and lifestyle factor associations with $\mathrm{CpG}$ island methylator phenotype and BRAF mutations in colon cancer. Int J Cancer. 2007;120(3): 656-663.

23. van den Brandt PA, Goldbohm RA, van 't Veer P, et al. A largescale prospective cohort study on diet and cancer in the Netherlands. J Clin Epidemiol. 1990;43(3):285-295.

24. Goldbohm RA, van den Brandt PA, Brants HA, et al. Validation of a dietary questionnaire used in a large-scale prospective cohort study on diet and cancer. Eur J Clin Nutr. 1994;48(4): 253-265.

25. Goldbohm RA, van 't Veer P, van den Brandt PA, et al. Reproducibility of a food frequency questionnaire and stability of dietary habits determined from five annually repeated measurements. Eur J Clin Nutr. 1995;49(6):420-429.

26. Van den Brandt PA, Schouten LJ, Goldbohm RA, et al. Development of a record linkage protocol for use in the Dutch
Cancer Registry for Epidemiological Research. Int J Epidemiol. 1990;19(3):553-558.

27. Goldbohm RA, van den Brandt PA, Dorant E. Estimation of the coverage of Dutch municipalities by cancer registries and PALGA based on hospital discharge data. Tijdschr Soc Gezondheidsz. 1994;72:80-84.

28. Hughes LA, Schouten LJ, Goldbohm RA, et al. Self-reported clothing size as a proxy measure for body size. Epidemiology. 2009;20(5):673-676.

29. Hettinger TH, Mueller BH, Gebhard H. Ermittlung des Arbeitsenergieumsatzes bei Dynamisch Muskulaerer Arbeit. Dortmund, Germany: Schriftenreihe der Bundesarbeit fur Arbeitsschutz; 1989.

30. Pylypchuk RD, Schouten LJ, Goldbohm RA, et al. Body mass index, height, and risk of lymphatic malignancies: a prospective cohort study. Am J Epidemiol. 2009;170(3):297-307.

31. Slattery ML, Potter JD. Physical activity and colon cancer: confounding or interaction? Med Sci Sports Exerc. 2002;34(6): 913-919.

32. de Vogel S, Bongaerts BW, Wouters KA, et al. Associations of dietary methyl donor intake with MLH1 promoter hypermethylation and related molecular phenotypes in sporadic colorectal cancer. Carcinogenesis. 2008;29(9):1765-1773.

33. Bolton-Smith C, Woodward M, Tunstall-Pedoe H, et al. Accuracy of the estimated prevalence of obesity from self reported height and weight in an adult Scottish population. J Epidemiol Community Health. 2000;54(2):143-148.

34. Nyholm M, Gullberg B, Merlo J, et al. The validity of obesity based on self-reported weight and height: implications for population studies. Obesity (Silver Spring). 2007;15(1):197-208.

35. Spencer EA, Appleby PN, Davey GK, et al. Validity of selfreported height and weight in 4808 EPIC-Oxford participants. Public Health Nutr. 2002;5(4):561-565.

36. Wada K, Tamakoshi K, Tsunekawa T, et al. Validity of selfreported height and weight in a Japanese workplace population. Int J Obes (Lond). 2005;29(9):1093-1099.

37. McKeown-Eyssen G. Epidemiology of colorectal cancer revisited: are serum triglycerides and/or plasma glucose associated with risk? Cancer Epidemiol Biomarkers Prev. 1994; 3(8):687-695.

38. Silvera SA, Jain M, Howe GR, et al. Energy balance and breast cancer risk: a prospective cohort study. Breast Cancer Res Treat. 2006;97(1):97-106.

39. Slattery ML, Caan BJ, Benson J, et al. Energy balance and rectal cancer: an evaluation of energy intake, energy expenditure, and body mass index. Nutr Cancer. 2003;46(2):166-171.

40. Okasha M, Gunnell D, Holly J, et al. Childhood growth and adult cancer. Best Pract Res Clin Endocrinol Metab. 2002; 16(2):225-241.

41. Engeland A, Tretli S, Austad G, et al. Height and body mass index in relation to colorectal and gallbladder cancer in two million Norwegian men and women. Cancer Causes Control. 2005;16(8):987-996.

42. Wei EK, Giovannucci E, Wu K, et al. Comparison of risk factors for colon and rectal cancer. Int J Cancer. 2004;108(3):433-442.

43. Hughes LA, van den Brandt PA, Goldbohm RA, et al. Childhood and adolescent energy restriction and subsequent colorectal cancer risk: results from the Netherlands Cohort Study. Int J Epidemiol. 2010;39(5):1333-1344.

44. Monteiro R, Azevedo I. Chronic inflammation in obesity and the metabolic syndrome. Mediators Inflamm. 2010;2010:289645. (doi:10.1155/2010/289645).

45. Elwing JE, Gao F, Davidson NO, et al. Type 2 diabetes mellitus: the impact on colorectal adenoma risk in women. Am J Gastroenterol. 2006;101(8):1866-1871. 
46. Giovannucci E, Michaud D. The role of obesity and related metabolic disturbances in cancers of the colon, prostate, and pancreas. Gastroenterology. 2007;132(6):2208-2225.

47. Hu FB, Manson JE, Liu S, et al. Prospective study of adult onset diabetes mellitus (type 2) and risk of colorectal cancer in women. J Natl Cancer Inst. 1999;91(6):542-547.
48. Jass JR. Classification of colorectal cancer based on correlation of clinical, morphological and molecular features. Histopathology. 2007;50(1):113-130.

49. Slattery ML, Wolff RK, Curtin K, et al. Colon tumor mutations and epigenetic changes associated with genetic polymorphism: insight into disease pathways. Mutat Res. 2009;660(1-2):12-21. 